\title{
Breast Cancer Series: Hormone Therapy ${ }^{1}$
}

\author{
Karen C. Daily and Martha C. Monroe ${ }^{2}$
}

Several drugs are used to block the estrogen receptor site or decrease the production of estrogen. Their use is called hormone therapy, endocrine therapy, or antiestrogen therapy. Use of these antiestrogen drugs helps many women reduce their risk of breast cancer. Both women who are at high risk of getting breast cancer and women who have had breast cancer may be prescribed one of these daily oral medications. These drugs can be used to prevent new breast cancer, to prevent recurrence of breast cancer in the breast or elsewhere in the body, or to control breast cancer that has already metastasized.

Most, but not all, breast tumors are sensitive to estrogen, which means they use estrogen to grow. These tumors are referred to as estrogen receptor/progesterone-receptorpositive tumors. Tumors that are not sensitive to estrogen (estrogen receptor/progesterone-receptor negative tumors) do not respond to antiestrogen hormone therapy. Hormone therapy drugs used in breast cancer prevention and treatment are very different from hormone replacement therapy, which is used by some women to help their symptoms during menopause. Hormone therapy drugs are more accurately described as antiestrogen treatments because they limit the amount of estrogen available to tumors.

Two drugs used as antiestrogens are tamoxifen and raloxifene $\left(\right.$ Evista $\left.^{\circledR}\right)$. They are members of a class of drugs called selective estrogen receptor modulators. The molecular structure of these drugs is similar to estrogen; therefore, they can fit into the estrogen receptor site on cancer cells. That site enables the cancer cell to take in estrogen and use the hormone to grow. Like a lock and key, when tamoxifen or raloxifene (the key) is stuck in the lock (the receptor), the door will not open and no other key (estrogen) can be used. Thus these drugs block estrogen from entering the cancer cell and slow down its growth.

Tamoxifen can be used by both premenopausal and postmenopausal women; raloxifene is approved by the Food and Drug Administration (FDA) only for postmenopausal women. Tamoxifen has rare but serious risks including an increased risk of blood clots and stroke, uterine cancer, and cataracts; raloxifene has a lower risk of these side effects. Less dangerous but more common side effects of both of these drugs include hot flashes and vaginal symptoms such as dryness or discharge. A beneficial side effect is a potential increase in bone density. Tamoxifen has been tested and used for over thirty years, allowing doctors to be confident that in many women the benefits of tamoxifen outweigh the risks. Raloxifene's FDA approved use is limited to prevention of breast cancer in women who have never been diagnosed with the disease and who are postmenopausal.

Aromatase inhibitors are a newer class of drugs prescribed only to postmenopausal women. Anastrozole (Arimidex ${ }^{\circledR}$ ) and Letrozole (Femara ${ }^{\circ}$ ) are FDA-approved for use for five years, or for two to three years following two to three years of tamoxifen to complete five years of treatment.

1. This document is FCS8836, one in a series of the Department of Family, Youth and Community Sciences, Institute of Food and Agricultural Sciences, University of Florida. Published June 2011. Visit the EDIS website at http://edis.ifas.ufl.edu.

2. Karen Daily is a Clinical Assistant Professor in Hematology \& Oncology and Martha Monroe is a Professor in the School of Forest Resources and Conservation. Both are at University of Florida.

The use of trade names in this publication is solely for the purpose of providing specific information. UF/IFAS does not guarantee or warranty the products named, and references to them in this publication do not signify our approval to the exclusion of other products of suitable composition. 
Exemestane (Aromasin ${ }^{\oplus}$ ) has FDA approval only for use following tamoxifen treatment. This group of medications prevents the production of estrogen by blocking the enzyme that converts androgens such as testosterone to estrogen. Conversion of testosterone to estrogen in fatty tissue is the main source of estrogen in postmenopausal women whose ovaries are no longer functioning. Aromatase inhibitors reduce bone density, which can lead to osteoporosis and increased risk of fractures. They also can cause muscle and bone aches. Hot flashes are associated with all of the antiestrogen agents and are most pronounced in younger women.

For an individual woman, assessment of menopausal status is very important in understanding the antiestrogen treatment options available to her. Menstrual history and age may provide sufficient information; however, hysterectomy or loss of menstrual periods with chemotherapy can complicate the determination of a woman's true menopausal status. Laboratory studies performed on blood samples measure amounts of follicle stimulating hormone (FSH) and estradiol (an estrogen), which can be useful in determining whether or not the ovaries have stopped producing estrogen.

An additional option available for postmenopausal women who have metastatic breast cancer is fulvestrant $\left(\right.$ Faslodex $\left.^{\otimes}\right)$. This drug also acts by blocking the estrogen receptor, but is administered by monthly intramuscular injection in a doctor's office. Like all of the antiestrogen treatments, it can cause hot flashes and other symptoms of menopause. Fulvestrant may be a useful option for women who have already received oral antiestrogen treatment.

There is not yet sufficient scientific evidence to know which option is best, but there is very strong evidence that some form of antiestrogen treatment after hormone receptor positive breast cancer in premenopausal women decreases the risk of recurrence. Options for these women include tamoxifen for five years or ovarian suppression in addition to either tamoxifen or an aromatase inhibitor for five years. Two methods of ovarian suppression most commonly used are oophorectomy (surgical removal of the ovaries) or monthly injectible medication such as Goserelin (Zoladex ${ }^{\circledast}$ ), a drug that chemically inactivates the ovaries temporarily. There has been some research that supports a longer duration of antiestrogen treatment for premenopausal women who become postmenopausal during the course of their treatment. This longer treatment would include tamoxifen for five years followed by an aromatase inhibitor for another five years. Premenopausal women who have not completed childbearing should discuss fertility preservation options with their physician.

\section{Summary}

- Women at high risk of developing breast cancer can reduce their risk by approximately $40 \%$ with antiestrogen therapy. Options are oral daily tablets of either tamoxifen (pre- or postmenopausal women) or raloxifene (postmenopausal women only) for five years.

- Women with hormone receptor (estrogen and/or progesterone)-positive tumors can cut their risk of recurrence after surgery in half with antiestrogen therapy. Options are tamoxifen, aromatase inhibitor (postmenopausal only), or tamoxifen followed by an aromatase inhibitor (postmenopausal only) oral daily tablets for five years.

- Tamoxifen is the only option for women who have functioning ovaries.

- Women with metastatic estrogen-receptor positive breast cancer can control the disease and delay the need for chemotherapy with antiestrogen therapy. This may include use of tamoxifen, an aromatase inhibitor, and/or fulvestrant injectible medication. 